greater influence of multinational corporations and neoliberal trade organisations.

Conclusion Following a realist re-synthesis of a large systematically collected dataset, three final programme theories were supported that offer an account of how the welfare state, political tradition and globalisation may exert an important effect on child and maternal health. Limitations include lack of stratification by level of development, contextual effects in interpreting fertility rates, and complexity in mapping political exposures to political parties internationally. Future realist analyses could consider other political exposures, such as governance and political capacity.

\section{P11 HOW DO PERINATAL RISK FACTORS MEDIATE THE RELATIONSHIP BETWEEN MATERNAL SOCIOECONOMIC STATUS AND PRETERM BIRTH? ANALYSIS OF ROUTINE HEALTHCARE DATA FROM THE NORTH WEST REGION OF ENGLAND}

${ }^{1}$ Philip McHale*, ${ }^{1}$ Daniela Schlüter, ${ }^{1}$ Ben Barr, ${ }^{2}$ Shantini Paranjothy, ${ }^{3}$ Angharad Care, ${ }^{1}$ David Taylor-Robinson. 'Department of Public Health, Policy and Systems, University of Liverpool, Liverpool, UK; ${ }^{2}$ Aberdeen Health Data Science Research Centre, University of Aberdeen, Aberdeen, UK; ${ }^{3}$ Centre for Women's Health Research, University of Liverpool, Liverpool, UK

\subsection{6/jech-2021-SSMabstracts. 101}

Background Prevalence of preterm birth (PTB) is increasing in England. Given significant inequalities in prevalence by maternal socioeconomic status (SES) and subsequent negative consequences on health and educational attainment for children, this represents a significant risk of widening population health inequalities. The aim is to understand how different exposures to risks mediate the relationship between SES and PTB.

Methods Analysis of routinely collected data for all births at Liverpool Women's Hospital between April 2009 and March 2020, covering an area of North West England with high levels of deprivation. The exposure was a dichotomised measure of maternal SES at birth (two most deprived Indices of Multiple Deprivation deciles compared to other eight deciles), and the outcome was PTB (gestational age $<37$ weeks) compared with term. Mediators were maternal smoking status, medical conditions, obstetric conditions, and BMI at booking. Covariates included maternal age at birth, method of labour onset, parity, baby sex, and previous PTB. Mediation was assessed using multivariable logistic regression for modelling the effect of SES on PTB after adjusting for covariates (minimally adjusted) with and without adjustment for the potential mediators and applying difference-of-coefficients method; the difference in odds ratio (OR) for SES between these models. Proportion mediated is $\left(\mathrm{OR}_{\text {mediator-adjusted - }}\right.$ $\left.\mathrm{OR}_{\text {minimally-adjusted }}\right) /\left(\mathrm{OR}_{\text {minimally-adjusted }}-1\right)$. All analysis was done in $\mathrm{R}$.

Results Analysis sample consisted of 81,680 births; $7.4 \%$ were preterm, while $64.1 \%$ were to low SES mothers. Of mothers, $19.1 \%$ were current smokers, $4.3 \%$ had medical conditions, $10.1 \%$ had obstetric conditions and $48.6 \%$ had healthy weight BMI. The OR for the effect of low SES on PTB in the minimally adjusted model was 1.26 (95\% CI 1.19-1.34). When mediators were included, OR reduced by $42 \%$ for smoking. OR for low SES did not change when maternal medical conditions or obstetric conditions were included, while OR increased when BMI was included (1.32, 95\% CI 1.22-1.44).

Discussion Our findings demonstrate that significant inequalities exist in the prevalence of PTB. The initial results from the mediation analysis suggest that smoking is partly explains these inequalities. Over half of this inequality is not explained by mediators included here. Limitations include unavailable data on other mediators (e.g. maternal mental health) and in the analysis approach. The next step is to analyse the data using the counterfactual approach to mediation. Reducing PTB is a priority for Maternity and Children's services, and the NHS' Long Term Plan. These results suggest that action on smoking during pregnancy will reduce inequalities.

\section{P12 INVESTIGATING CAUSALITY BETWEEN ADIPOSITY AND WOMEN'S REPRODUCTIVE FACTORS: A MENDELIAN RANDOMIZATION ANALYSIS}

Claire Prince*, Gemma C Sharp, Laura D Howe, Abigail Fraser, Rebecca C Richmond. MRC Integrative Epidemiology Unit, Bristol Medical School, University of Bristol, Bristol, UK

\subsection{6/jech-2021-SSMabstracts. 102}

Background Reproductive factors (RFs) in women, such as menarche and menopause, the age at which women start and stop having children, and the number of children they have, have been implicating in disease risk including, breast cancer, cardiovascular, respiratory diseases. While body mass index (BMI) has similarly been identified as a risk factor in such diseases. Studies have identified strong bidirectional associations between adulthood BMI and RFs in women, and higher body size in girls was reported to be associated with earlier age at menarche and menopause. Considering the evidence of interplay between BMI and RFs, it is important to consider causal relationships which may underlie these associations. This study aims to appraise causality between both childhood and adulthood adiposity and the RFs, as well as establishing the magnitude of any identified relationships and direction of causality.

Methods We used data from the UK Biobank and genetic consortia with data available for RFs and BMI. We then applied Mendelian randomization (MR) methods to estimate the causal relationships between RFs and BMI both in childhood and adulthood. Sensitivity analyses were used to investigate directionality of the effect observed, to test for evidence of pleiotropy and to account for any sample overlap.

Results MR revealed body size and BMI in childhood and adulthood respectively have potentially causal relationships with a number of RFs, as well as a number of reproductive having a potentially effect on BMI in adulthood. Notably higher body size during childhood appears to lead to an earlier age at menarche $(\operatorname{Beta}(\mathrm{B})=-0.65,95 \%$ confidence intervals $(\mathrm{CI})=-0.74,-0.56)$, age at first, $(B=-0.07, C I=-0.12$, $0.01)$ and last birth $(B=-0.09, C I=-0.13,-0.04)$. While higher BMI during adulthood seems to lead to an earlier age at menopause $(B=-0.53, C I=-0.82,-0.23)$, age at first live birth $(B=-$ $1.72, \mathrm{CI}=-2.06,-1.39)$, age at last live birth $(\mathrm{B}=-1.40, \mathrm{CI}=-$ $1.68,-1.13)$, earlier age at first sexual intercourse $(B=-1.09$, $\mathrm{CI}=-1.39,-0.78)$ and increased likelihood of having children $(\mathrm{OR}=1.11, \mathrm{CI}=1.01,1.21)$. Finally, findings suggests that earlier menarche $(B=-0.03, C I=-0.04,-0.03)$ and age at first birth $(B=-0.05, C I=-0.06,-0.03)$ and age first had sexual 
intercourse $(\mathrm{B}=-0.03, \mathrm{CI}=-0.04,-0.01)$ may lead to higher adulthood BMI.

Conclusion The findings of this study present strong evidence for causal interplay between adiposity and women's RFs and future studies should consider adiposity when investigating risk of RFs, whether as a potential confounder or mediator, on disease risk.

\section{P13 HOW HAVE UK MATERNAL HEALTH SERVICES CHANGE IN RESPONSE TO THE COVID-19 PANDEMIC? - A SYSTEMATIC REVIEW}

${ }^{1}$ Hannah Beetham*, ${ }^{2}$ Lisa Hurt, ${ }^{3}$ Lauren Copeland. 'School of Medicine, Cardiff University, Cardiff, UK; ${ }^{2}$ Division of Population Medicine, Cardiff University, Cardiff, UK; ${ }^{3}$ DECIPHer School of Social Sciences, Cardiff University, Cardiff, UK

10.1136/jech-2021-SSMabstracts. 103

Rationale Many expectant and new mothers have been impacted by the restructuring of maternal services that has occurred in response to the pandemic. Although there is hopefully an end in sight, restrictions may continue for some months. It is therefore important to assess the changes that have occurred, in order to prevent further harm to mothers, or to realise and implement any positive changes permanently. Aim: To analyse the different ways in which antenatal, obstetric, and postnatal services in the UK have adapted to the COVID-19 pandemic.

Methods Key-word searches were used to systematically scope three databases for papers that reported on any aspect of maternal care or services and COVID-19 until 03/12/2020. In total, 2,299 papers' titles and abstracts were screened according to pre-set eligibility criteria. Half were double-screened by a second researcher, and discrepancies were discussed between three researchers until resolved. Full-text screening $(n=344)$ excluded papers that weren't relevant, or didn't include primary data. Excluded papers and papers that the lead researcher flagged for a second opinion were checked by a second researcher. At this point, the decision was made to focus on UK services, due to the high number of papers. Next steps will be to extract data from the final sample of papers $(n=12)$ and appraise their quality. Narrative synthesis will form a coherent report on how services in the UK have changed.

Results Preliminary results show that there have been shifts in some maternal services towards virtual delivery. Additionally, there has been a change in the number of antenatal and postnatal appointments, including the number and nature of antenatal emergency presentations. There has also been a reduction in the care choices available to pregnant women, including changes to birth plans. Furthermore, there have been changes to obstetric unit layouts and care delivery, including changes to the rates of C-sections and general anaesthesia. Additional results will be submitted before the June 28th.

Population Health Relevance This review is expected to highlight some interesting changes that have occurred in UK maternal services since the beginning of the pandemic. Some may have changed maternal services for the better, while others may highlight some difficulties that new mothers have faced, and will be important to address in case of continuing restrictions or future pandemics. Further research will focus on qualitatively assessing the experiences of new mothers during the pandemic, to gain first-hand insights into how they were affected by the changes.

\section{P14 NEO-MALTHUSIANISM IN THE CONCEPTION OF THE FAMILY PLANNING FRAME: THE ROLE OF THE FORD FOUNDATION IN POPULATION CONTROL IN CHINA AND INDIA}

${ }^{3}$ Tiasangla Longkumer*. ' Centre of Social Medicine and Community Health, Jawaharlal Nehru University, Delhi, India; ${ }^{2}$ Harvard Yenching Institute, Harvard University, Cambridge, USA; ${ }^{3}$ Institute of Chinese Studies, Think Tank, Delhi, India

\subsection{6/jech-2021-SSMabstracts. 104}

Background The second half of the 20th century experienced the rise of demography as a discipline with huge outpouring of technical aid and resources to curb the population of the developing nations with a special focus on Asia. China and India figured in the imagination of these demographers who believed Neo Malthusian theory of positive checks on population control. The theory of Neo Malthusianism took precedence among senior technocrats, demographers and government officials in the 1950s and 60s. China experienced the peak of Neo Malthusianism with the one-child policy in the late 1970s and India with the Emergency in the early 1970s.

Methods Tracing the conceptual understanding of Neo-Malthusianism which was the theoretical backbone of Ford's population control programmes, this paper would encompass the historical development of the Family Planning program in independent India and socialist China with a sight on the various important actors involved, the geopolitical context of Ford's entry, strategies adopted and the implications of Family planning on the health systems.Using archival research, secondary literatures and key informant interviews, this study adopts a qualitative method and aims to study the efforts of the Ford Foundation to promote, advance and institutionalize population control through the Family Planning program in China and India.

Data The study primarily draws on archival data namely government correspondence, memoranda, notes, reports, personal papers of some of the important actors, minutes of meetings, manuscripts of memoirs and autobiographies, reports of committees and commissions, working papers, theses and secondary references. Archival research was conducted at the Rockefeller Archive Centre, Harvard University Archives, Columbia University Ford Foundation collections online. Secondary literatures particularly the writings of John C. Caldwell, Gyan Prakash, Dwight Macdonald, Mohan Rao, Matthew Connelly, Susan Greenhalgh and several others have been consulted. Key informants included Dr. Lincoln Chen and Dr. Shirish Kavadi.

Discussion By the early 1950s, the growing population of China and India became a source of high concern in the west and the eastern elites. Complementary to the voiced concern for growing number of Chinese and Indians, another important precursor of the Ford Foundation efforts was the changing political landscape. The formation of the Marshall plan, India's action towards curbing population growth in the 1950s and recommendations of the 1948 field trip in the Far East induced Ford's population control activities and changed the discourse of population health in China and India. 